\title{
THE SIGNIFICANCE OF DEFECTS IN NICKEL BASE SUPERALLOYS
}

\author{
S.R. Holdsworth \\ GEC Turbine Generators Limited \\ Whetstone, Leicester LE8 3LH., U.K.
}

The paper reviews the types of metallurgical flaw which may exist in nickel base alloy components used in industrial gas turbine plant. The dimensions of these defects which may be detected and sized by non-destructive examination are then compared with those allowed by fracture mechanics calculations using data generated in the author's laboratory.

\section{INTRODUCTION}

A high degree of care is taken in the melting, casting, working and inspection of nickel base alloys for gas turbine parts, thus maintaining the number and size of metallurgical defects to a minimum. This effort is not surprising since the majority of these materials were initially developed for aero-engines which have always demanded the highest integrity in terms of performance, reliability and safety.

In the wake of their aeronautical counterparts, the manufacturers of industrial gas turbines have adopted many of the same nickel base alloys and their standards. However, the increase in size of industrial sets has rapidly outpaced that of vehicular units and materials developed for essentially small scale pieces are now being adopted for much larger compononts.

A review of the application of nickel base alloys within the power generation industry has revealed that, as ruling sections have increased, the difticulties experienced during melting, casting and working operations and consequently the probability of components containing greater numbers of larger defects have also increased. Furthermore with increasing ruling section, nickel base alloy components have become less amenable to internal non-destructive examination. 
This paper reviews the sizes of metallurgical defects detectable in larger nickel base alloy components and compares them with some allowable defect sizes determined using basic fracture mechanics calculations and laboratory data generated by the author.

\section{TYPES OF DEFECTS}

There are several forms of metallurgical flaw which may exist in nickel base components. Some are unique to the material type and the remainder are characteristic of the fabrication route adopted.

Nickel base alloys exhibit low thermal conductivities, moderately wide solidification ranges and high shrinkage during solidification. As a result they are prone to coarse grain structures, extensive macro and micro segregation, high internal stresses, and hot tearing, particularly as ingot and casting sizes increase. Microsegregation can generally be eliminated by working or homogenisation, but macrosegregation can rarely be totally removed. The local concentrations of solute elements are accompanied by the precipitation of primary carbide and in severe cases agglomerations of coarse carbides. Contraction during cooling between liquidus and solidus is typically 2 to 3 percent in nickel base alloys and without suitable precautions these materials exhibit a high susceptibility to hot tearing and a range of shrinkage defects.

Gaseous products are also responsible for the presence of defects such as porosity and entrapped oxide although these materials are probably more resistant than most to this type of flaw because of the tendency to adopt vacuum melting and casting procedures. Nevertheless, these procedures do not eliminate the formation of brittle nitride and carbonitride agglomerates and stringers. The nickel base alloys are particularly susceptible to these defects since they tend to contain significant proportions of elements such as $\mathrm{Cr}, \mathrm{Ti}, \mathrm{Al}$, $\mathrm{Nb}$, and $\mathrm{V}$ which reduce the activity of nitrogen and its effective removal by vacuum.

\section{DETECTION AND SIZING}

A number of techniques are available for the nondestructive detection of surface and embedded defects in nickel base alloys. The minimum sizes which can be identified by these methods in practical circumstances are estimated bclow. 
The detection of surface defects in forgings and castings is conducted visually. Chemical etching reveals the presence of stringer type inclusions (e.g. TiCN), segregate, shrinkage and porosity. Fluorescent penetrate dye techniques simplify inspection and enable detection of all but the tighlest planar flaws. In a production environment the minimum defect length which may be readily detected is approximately $0.4 \mathrm{~mm}$ but this dimension is dependent on the scope of the examination, oxide content and crack opening. Cracks with openings of less than around $0.5 \mu \mathrm{m}$, for example, are rarely revealed by fluorescent penetrant dye.

Surface defects and embedded defects close to the surface in tube and bar products can be detected using eddy current techniques. This method is capable of detecting axial defects approximate? $0.1 \mathrm{~mm}$ deep, provided their length is greater than $10 \mathrm{~mm}$, but it is susceptible to missing circumferential cracks.

Embedded defects are detected using ultrasonic or radiographic methods. Nickel base alloys, as-cast, are prone to coarse grain structures and defects having comparable dimensions to the grain size can be easily missed using ultrasonics because of wave scattering and attenuation. This method is, therefore, rarely adopted for castings, especially large ones. Grain size reduction as a result of working makes wrought components more amenable to ultrasonic examination provided it is conducted before ageing. Nevertheless, the level of detectability varies with chemistry and ruling section. Defects, $0.5 \mathrm{~mm}$ diameter, are detectable in small forgings but this dimension increases to $1 \mathrm{~mm}-2 \mathrm{~mm}$ diameter and on to $3 \mathrm{~mm}-4 \mathrm{~mm}$ diameter in $150 \mathrm{~mm}$ and $250 \mathrm{~mm}$ thick disc cheeses respectively. (ref.1). In general, radiography is only used for the examination of castings, the technique being capable of assessing pieces up to $450 \mathrm{~mm}$ thickness. In practical circumstances the limit of detectability for volumetric defects increases from around $0.1 \mathrm{~mm}$ to $1.0 \mathrm{~mm}$ with increasing thickness from $5 \mathrm{~mm}$ to 150mm (Rer. 2). The minimum size for planar derects is greater, depending on tightness and orientation.

\section{ALLOWABLE DEFECT SIZES}

In recent years fracture mechanics methodology has been increasingly adopted by the power generation industry as a means of predicting allowable defect sizes. In a simple analysis three defect sizes are identified to predict cllowable dimensions. These are the critical, final and initial defect sizes (Figure 1 ). 


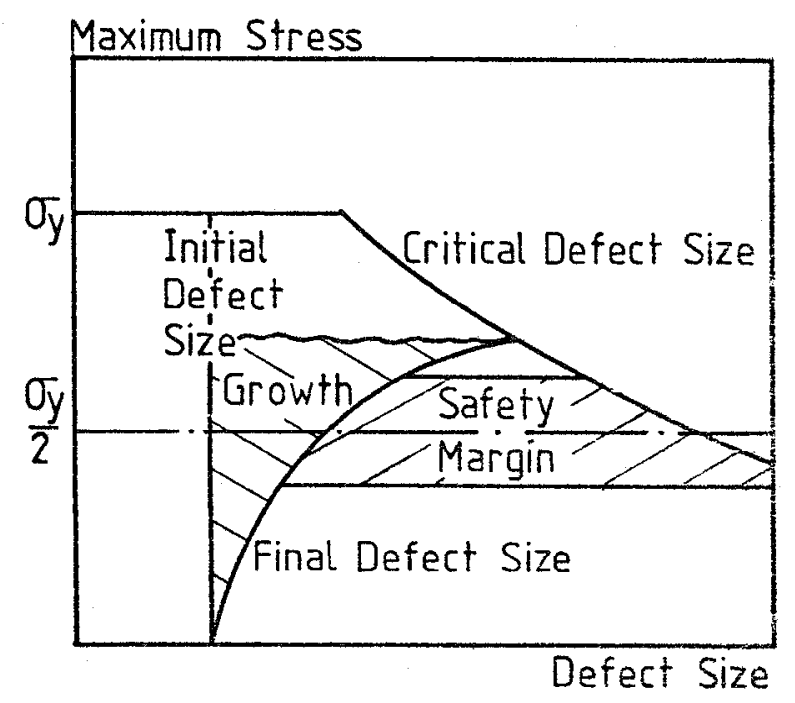

\section{Figure.1. Allowable Defect Sizes}

The critical derect size is that dimension above which unstable fast fracture occurs. The calculation of critical defect size is well documented, the main data requirement being the material's fracture toughness $\left(K_{1 C}\right)$. $K_{I c}$, the critical value of $K_{1}$ (Refs. 3,4 ), is determined directly on laboratory specimens having precrack lengths and thicknesses of a sufficient size to satisfy the linear elastic requiremonts of ASTM(5) or indirectly by correlation with arr elasticplastic parameter such as $J_{\perp C}$ (Ref. 6) using sub-size testpieces. The determination by correlation is the more common since only the largest components are of a sufficient size to yield valid ASTM specimens. The fracture toughnesses of a number of nickel base alloys are summarised in Table 1.

Unstable failures cannot bo tolorated in gas turbine plant. A final defect size which must not be exceeded during the design life is therefore defined as some fraction of the critical defect size (Figure 1).

The initial defect size which is allowed in a component entering service is that size which will not extend beyond the final defect size by some sub-critical growth mechanism during the design life (Figure 1). In gas turbine components the driving forces for stable crack propagation include mechanical and thermal fatigue, creep and corrosion. The mathematical prediction of allowable initial defect sizes is based on the measurement of growth rates induced in laboratory 
Table 1. Fracture Toughness Values for Some Nickel Base Alloys

\begin{tabular}{|c|c|c|c|c|}
\hline Material & $\begin{array}{c}\text { Yield } \\
\text { Strength } \\
\text { (MPa) }\end{array}$ & $\begin{array}{c}\text { Testpiece } \\
\text { Thickness } \\
(\mathrm{mm})\end{array}$ & $\begin{array}{c}\mathrm{K}_{\mathrm{c}} \\
\left.\text { (MPam }^{1 / 2}\right)\end{array}$ & $\begin{array}{c}\mathrm{K}_{\text {ic }} \\
\left.\text { (MPam }^{1 / 2}\right)\end{array}$ \\
\hline Nimonic 90 & 780 & 12.7 & 105 & \\
Nimonic 901 & 900 & 76.2 & 167 & 12 \\
& & 25.4 & 124 & \\
Inconel 718 & 1200 & 76.2 & 160 & $(160)$ \\
Nimocast 713LC & 750 & 25.4 & & 90 \\
Nimocast 738LC & 850 & 25.4 & & 73 \\
Nimocast 792 & 1000 & 25.4 & & 88 \\
Nimocast 739 & 800 & 25.4 & & 71 \\
& & & & \\
\hline
\end{tabular}

Table 2. Comparison of Sireable and Allowable Defect Dimensions

\begin{tabular}{|c|c|c|c|c|c|}
\hline \multirow[b]{2}{*}{ Defect Type } & \multirow{2}{*}{$\begin{array}{l}\text { Section } \\
\text { Size } \\
(\mathrm{mm})\end{array}$} & \multicolumn{4}{|c|}{ Defect Diameter (mm) } \\
\hline & & $\begin{array}{l}\text { Detect- } \\
\text { able }\end{array}$ & Initial & Final & Critical \\
\hline $\begin{array}{l}\text { Nimonic } 901 \\
\text { semi-circular } \\
\text { surface } \\
\text { embedded penny } \\
\text { Inconel } 718 \\
\text { semi-circular } \\
\text { surface } \\
\text { embedded penny } \\
\text { Nimocast 738LC } \\
\text { semi-circular } \\
\text { purface } \\
\text { embedded penny }\end{array}$ & $\begin{array}{l}150 \\
250\end{array}$ & $\begin{array}{l}\sim 0.4 \\
1-2 \\
3-4\end{array}$ & $\begin{array}{l}5.5^{*} \\
6.8^{*}\end{array}$ & $\begin{array}{l}31.8 \\
39.8\end{array}$ & $\begin{array}{l}159.0 \\
199.0\end{array}$ \\
\hline
\end{tabular}

* determined using $\triangle K_{0}$ (Figure 2) 
precracked specimens, subject to conditions reproducing the in-service driving forces. The author has measured growth rates and threshold $\Delta$ Ko values for a number of nickel base alloys operating at ambient (Figure 2) and elevated temperatures (Figure 3) in laboratory air. To date only a limited volume of data exists for crack growth in industrial gas turbine environments. This evidence suggests that fatigue crack growth rates in the presence of oxygen or sulphur bearing species can be significantly faster than those in air (Ref. 7).

Using the laboratory data given in Table 1 and Figure 2 pessimistic allowable sizes of (a) semi-circular surface defects and (b) embedded penny-shaped defects in two nickel base forgings and a superalloy casting are determined assuming a critical to final defect size ratio of five. The sizes given in Table 2 assume maximum applied tensile stress levels of half yield strength magnitude, acting normal to the plane of the defect, and design lives of $10^{4}$ cycles for the forgings and $10^{10}$ cycles for the casting.

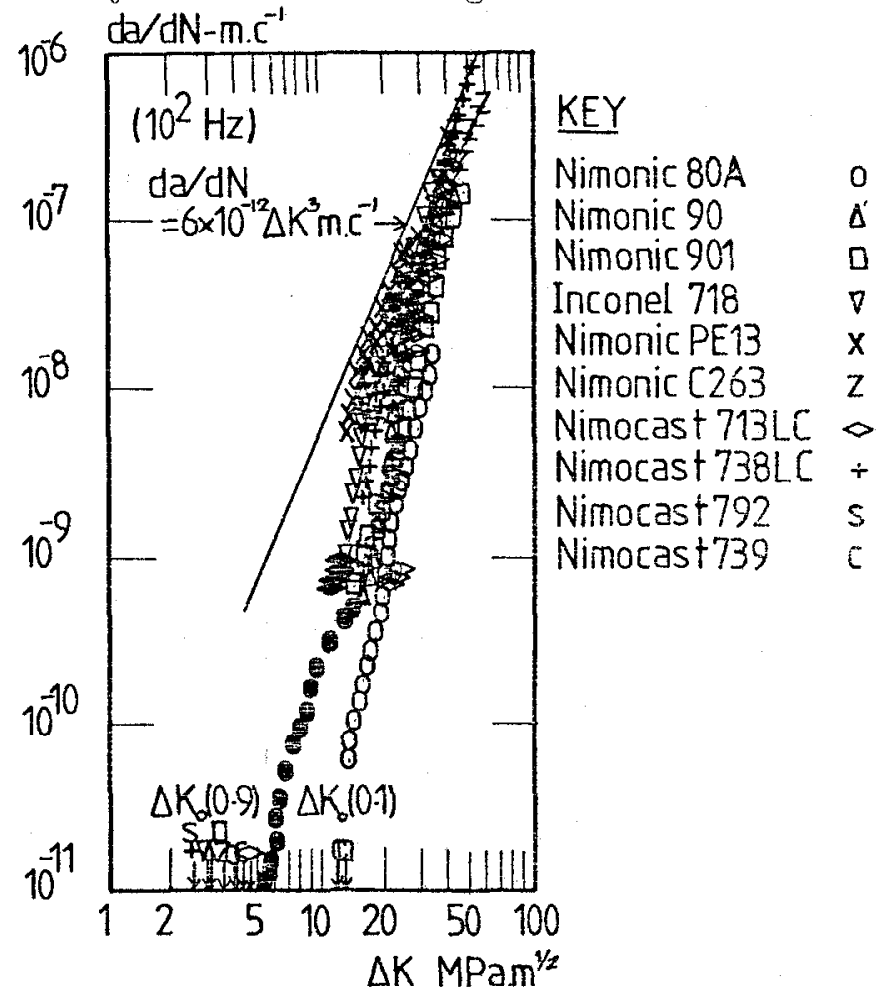

Figure.2. High Cycle Fatigue Crack Growth in a Range of Nickel Base Alloys at Ambient Temperature. 


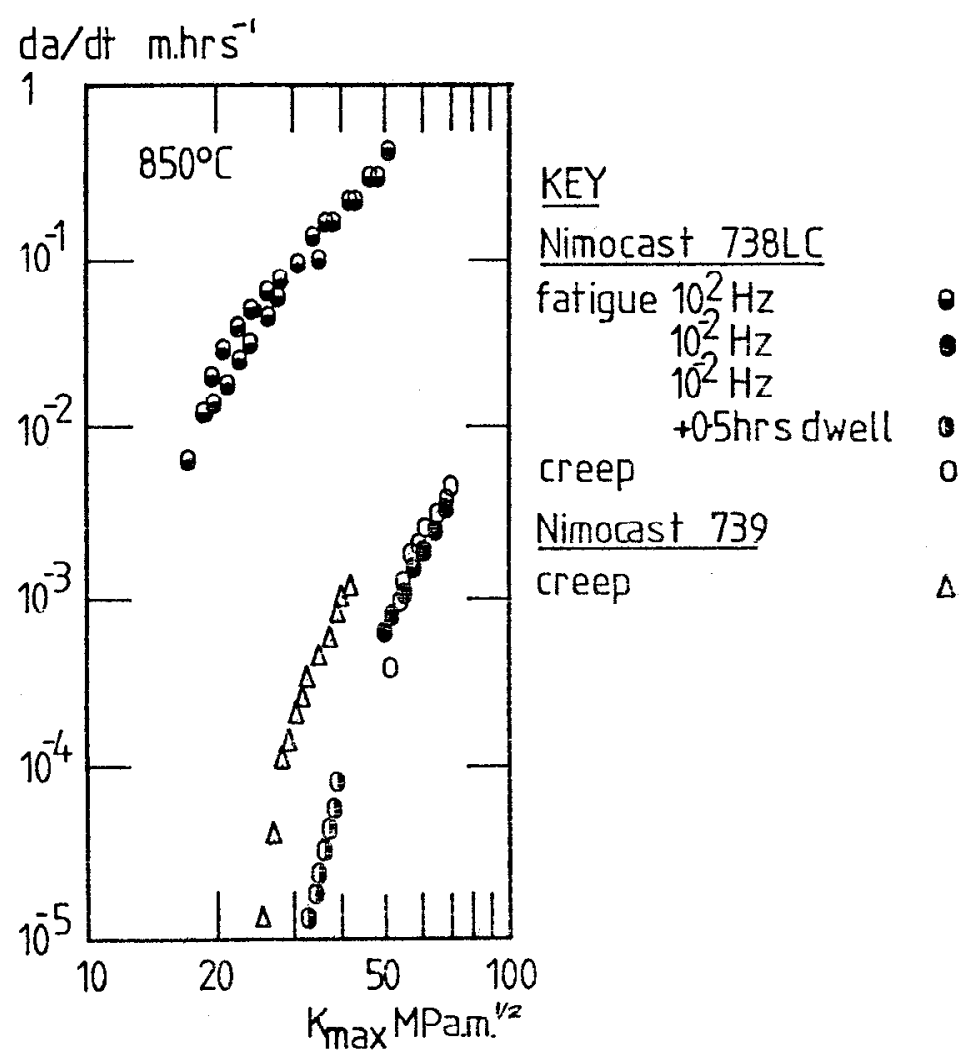

Figure 3 Elevated Temperature Crack Propagation

\section{DEFECT SIGNIFICANCE}

The cases chosen in the prccoding scction, in many ways resemble large disc forgings and blade castings in modern highly rated machines, and represent two examples of components manufactured from nickel base alloys which were originally developed for equivalent parts, of a smaller scale, in aeroengines. It is evident from Table 2 that the allowable initial surface defect sizes calculated are at least a factor of five greater than the limits of detectability offered by current surface inspection techniques for all but the tightest flaws. A similar situation also exists for embedded defects located in tough, medium strength nickel base forgings. However, if materials of higher strength and/or lower tough- 
ness, and/or ruling sections of greater size are adopted, the degree of confidence in detecting and sizing allowable embedded defects diminishes to unacceptable limits in certain circumstances.

\section{CONCLUDING REMARKS}

A comparison of the dimensional limits for defect sizes, offered by a range of non-destructive inspection methods, with some simple pessimistic allowable defect sizes, has emphasised the need for care in the adoption of certain nickel base alloys for larger scale industrial gas turbine components. In their current state of development this range of materials are more likely to contain significant, defects and be less amenable to internal non-destructive examination as ruling section sizes increase. While the margin of safety between sizeable and allowable internal defect dimensions in medium strength, high toughness forgings is acceptable, it is clearly a cause for concern in higher strength and/or lower toughness alloys being adopted for heavier castings and forgings than those for which they were originally developed, particularly if their design criteria are based on yield strength alone.

\section{ACKNOWLEDGEMENTS}

The author wishes to thank GEC Gas Turbines Limited for permission to reproduce these results and to acknowledge the valuable discussions with Dr. P. Greenfield, Mr. J. Davies and other colleagues in the Central Metallurgical Laboratories.

\section{REFERENCES}

1. Private discussions with a number of British Forgemasters.

2. Quality Technology Handbook, and Edition. NDT Contre, Harwell, IPC Science and Techology Press, 1975.

3. "Method for $k_{i}$ Determination". ASME Sections XI Division 1 (1977), Article A-3000.

4. D.P. Rooke and D.J. Cartwright. "Compendium of Stress Intensity Factors", HMSO, 1976. 
5. "Standard Method of Test for Plane-Strain Practure Toughness of Metallic Materials". ASTM E399-78, 1978.

6. G.A. Clarke et al. "A Procedure for the Determination of Ductile Fracture Toughness Values using J Integral Techniques". Journal of Testing and Evaluation, JTEVA, v7, n1, January 1979, pp 49-56.

7. S. Floreen and R.H. Kane, "Effects of Environment on High Temperature Fatigue Crack Growth in a Superalloy", Metallurgical Transactions A, v10A, November 1979, 1745. 